\section{OC52 A RETROSPECTIVE STUDY OF MYELOID LEUKAEMIA IN CHILDREN WITH DOWN SYNDROME IN IRELAND}

${ }^{1}$ Gavin P Dowling*, ${ }^{2,3}$ Andrea Piccin, ${ }^{2}$ Katherine T Gavin, ${ }^{2,1}$ Aengus O'Marcaigh, ${ }^{2,4}$ Owen P Smith. 'Royal College of Surgeons in Ireland, Dublin, Ireland; ${ }^{2}$ Our Lady's Children's Hospital Crumlin, Dublin, Ireland; ${ }^{3}$ Medical University of Innsbruck, Innsbruck, Austria; ${ }^{4}$ University College Dublin (UCD), Dublin, Ireland

\subsection{6/archdischild-2019-epa.49}

Background Haematological abnormalities are common in children with trisomy 21 . These children have a remarkably high risk of acute leukaemia. The incidence of acute myeloid leukaemia is 150 fold greater in young children with DS compared to children of the same age without DS. Acute Megakaryoblastic Leukaemia (AMKL) is a subtype of myeloid leukaemia and is the most common leukaemia type in children with DS under 4 years of age. AMKL is often preceded by a transient neonatal pre-leukaemic syndrome, called Transient Myeloproliferative Disorder (TMD). Although TMD often spontaneously resolves, $20-30 \%$ of these patients subsequently develop AMKL within the first 4 years of life.

Aims To perform a retrospective consecutive national audit of all documented cases of childhood TMD and AMKL-DS from 1990-2018 at the National Paediatric Haematology/Oncology Centre, Our Lady's Children's Hospital Crumlin (OLCHC) Ireland. We also aimed to compare our demographic and outcome findings with the recent medical literature and make recommendations for future research.

Methods Charts of the patients with a diagnosis of AMKL and treated consecutively at (OLCHC) between $1990-2018$ were sourced from medical records. Charts and the hospital database were reviewed and information including date and age of diagnosis of AML, comorbidities, previous history of TMD, treatment regime and outcome was noted. The Hospital Patient Administration System (PAS) was interrogated for additional information such as percentage blasts in the bone marrow and blood count values at time of diagnosis of AMKL. Cytogenetic information was obtained by accessing a dedicated database. Kaplan-Meier survival curves were constructed.

Results Twenty-seven patients with AMKL-DS were treated in OLCHC. A prior neonatal diagnosis of TMD was described in 10 patients $(37 \%)$. Patients had a low platelet count (median $\left.34 \times 10^{9} / \mathrm{L}\right)$ at presentation. Nineteen patients $(70 \%)$ are alive and well, in complete remission, at a median follow up of 11.4 years. Overall survival (OS) of this cohort has risen from $54 \%$ from those treated between the years $1990-2004(\mathrm{n}=$ 13) to $93 \%$ for those treated between the years $2005-2018$ $(\mathrm{n}=14)$.

Conclusion High cure rates are observed in AMKL-DS using current polychemotherapy protocols. However, in general children with DS are more prone to the cytotoxic effects of chemotherapy, which can lead to significant treatment related mortality. The finding of a low platelet count at time of diagnosis is in keeping with the knowledge that AMKL-DS is a malignancy of platelet progenitor cells.

\section{OC53 114 CASES OF CHRONIC GRANULOMATOUS DISEASE IN MAINLAND CHINA}

Liping Jiang*, Shiyu Wang. Children's Hospital of Chongqing Medical University, Chongqing, China

10.1136/archdischild-2019-epa.50
Background Chronic granulomatous disease (CGD) is a rare inherited disorder, with phagocytes failing to produce antimicrobial superoxide due to deficient NADPH oxidase activity. Mutations in the gene encoding CYBB are responsible for the majority of the CGD cases.In this study, we described the clinical and molecular features of 114 suspected CGD patients in mainland China.

Methods During 2013 to 2018, we enrolled 114 cases diagnosed with CGD in Children's Hospital of Chongqing Medical University. Patients' clinical data were collected, neutrophil function was detected, and the genotype of patients and fetal cells in the carrier mother's amniotic fluid were detected.

Results 114 CGD patients (109 male, 5 female), from 20 provinces of mainland China. The mean age at onset was 4 months, and the mean age of diagnosis was 23.1 months. To date, 33 patients have died, 27 patients have hemopoietic stem cell transplantation, and 12 patients were lost. For all patients, the types of genetic mutations include CYBB (95, 83.3\%), CYBA (8), NCF1 (3) and NCF2 (2), and 6 cases have not been found yet. The type of infection symptoms in this cohort included recurrent fever (100\%), pneumonia $(92$. $1 \%)$, tuberculosis $(58.8 \%)$, BCG disease $(55.1 \% ; 54 / 98)$, repeated diarrhea $(45.6 \%)$ and others. In addition, we prenatally diagnosed 8 XL-CGD, 6 healthy fetuses and 1 carrier (a XL-CGD female infant patient with X-chromosome inactivated).

Conclusions Children with recurrent bacterial and fungal infection not easy to cure, with higher inflammatory markers, with or without BCG disease and family history, should be alert to CGD. BCG disease and tuberculosis is still a very severe problem in CGD patients in China. Early diagnosis and prophylaxis treatment can prolong the survival time of CGD patients. DHR-1,2,3 test and NBT test can quickly aid in the diagnosis of CGD. Molecular analysis is an important tool for identifying diagnosis, prenatal diagnosis of CGD.

\section{OC54 INTRAOPERATIVE NERVE INTEGRITY MONITOR AS PART OF SAFETY DISSECTIONS OF RECURRENT LARYNGEAL NERVE IN CHILD AND ADOLESCENTS THYROID SURGERY}

Anatoly Romanchishen*, Filipp Romanchishen, Igor Karpatsky, Kristina Vabalayte. St Petersburg State Pediatric Medical University, St. Petersburg, Russian Federation

\subsection{6/archdischild-2019-epa.51}

Introduction In thyroid cancer (TC) operations most frequently damaged vital structures are recurrent laryngeal nerves (RLN), external branch of superior laryngeal nerves and parathyroid glands.

Material and methods To improve our knowledge of RLN surgical anatomy we investigated autopsy material (30 RLN), photo documented 1717 RLNs. There are about 30 variations of RLN and inferior thyroid artery attitude and three most common points of RLN visualization They are: 1 - subclavial point known as Lore's triangle, 2 - RLN 'cross point' with the inferior thyroid artery and 3 - RLN laryngeal entry point. After anatomical investigationswe used the Intraoperative Nerve Integrity Monitor (IONIM) during thyroid operations (2001-2016) in adults (1791), adolescents and children (117 cases).

Results It was found that RLNs in $3^{\text {rd }}$ point were crossed by blood vessels in 94.9\% (Romanchishen F.A., 2006). Ligation 